\title{
Are there distinct subtypes in Tourette syndrome? Pure-Tourette syndrome versus Tourette syndrome-plus, and simple versus complex tics
}

This article was published in the following Dove Press journal:

Neuropsychiatric Disease and Treatment

II June 2015

Number of times this article has been viewed

\author{
Valsamma Eapen ${ }^{1,2}$ \\ Mary M Robertson 3,4 \\ 'School of Psychiatry, University \\ of New South Wales, Sydney, NSW, \\ Australia; ${ }^{2}$ Academic Unit of Child \\ Psychiatry, South Western Sydney \\ Local Health District, Ingham \\ Institute, Liverpool, NSW, Australia; \\ ${ }^{3}$ Neuropsychiatry, University College \\ London, UK; ${ }^{4}$ St Georges Hospital \\ and Medical School, London, UK
}

\begin{abstract}
This study addressed several questions relating to the core features of Tourette syndrome (TS) including in particular coprolalia (involuntary utterance of obscene words) and copropraxia (involuntary and inappropriate rude gesturing). A cohort of $400 \mathrm{TS}$ patients was investigated. We observed that coprolalia occurred in 39\% of the full cohort of 400 patients and copropraxia occurred in $20 \%$ of the cohort. Those with coprolalia had significantly higher Yale Global Tic Severity Scale (YGTSS) and Diagnostic Confidence Index (DCI) total scores and a significantly higher proportion also experienced copropraxia and echolalia. A subgroup of 222 TS patients with full comorbidity data available were also compared based on whether they had pure-TS (motor and vocal tics only) or associated comorbidities and co-existent psychopathologies (TS-plus). Pure-TS and TS-plus groups were compared across a number of characteristics including TS severity, associated clinical features, and family history. In this subgroup, $13.5 \%$ had pure-TS, while the remainder had comorbidities and psychopathologies consistent with TS-plus. Thirty-nine percent of the TS-plus group displayed coprolalia, compared to $(0 \%)$ of the pure-TS group and the difference in proportions was statistically significant. The only other significant difference found between the two groups was that pure-TS was associated with no family history of obsessive compulsive disorder which is an interesting finding that may suggest that additional genes or environmental factors may be at play when TS is associated with comorbidities. Finally, differences between individuals with simple versus complex vocal/motor tics were evaluated. Results indicated that individuals with complex motor/vocal tics were significantly more likely to report premonitory urges/sensations than individuals with simple tics and TS. The implications of these findings for the assessment and understanding of TS are discussed.
\end{abstract}

Keywords: Tourette syndrome, comorbidity, simplex tics, complex tics

\section{Introduction}

Tourette syndrome (TS) is a childhood onset neuropsychiatric movement disorder characterized by multiple motor tics and one or more vocal/phonic tics. ${ }^{1,2}$ A duration of more than 1 year since the first tic and an onset before 18 years is also required for diagnosis. ${ }^{1,2}$ TS is associated with both simple and complex tics. Simple motor tics are restricted to a small group of muscles in the body and simple vocal tics to small sounds including throat clearing or sniffing. ${ }^{3}$ Complex tics involve several muscle groups or purposeful movements including touching or sniffing objects as well as the use of words or phrases. Complex tics can include echolalia (repeated vocalizations), palilalia (repetition of words or phrases), echopraxia (repeated actions), palipraxia (repeating the last act), self-injurious behaviors, complex vocalizations (eg, animal sounds), coprolalia (swearing), copropraxia (inappropriate touching) etc. ${ }^{4}$ Other associated features include 
non-obscene socially inappropriate behaviors and remarks, concern for symmetry and "evening up", having to do things "just right" and forced touching etc which can be regarded as a complex tic (mostly involuntary with little or no warning) or an "impulsion" (resulting from poor impulse control and the person may experience pleasure or gratification in some instances) or a compulsive behavior (preceded by an obsessional thought or a compulsive urge).

Although only motor and vocal tics are necessary for a diagnosis of TS, the incidence of tics in the absence of other associated features and comorbidities occurs in only around $10 \%$ of cases (pure-TS) while the remainder have a number of associated comorbidities (TS-plus). ${ }^{5,6}$ In this regard, TS has been commonly associated with comorbid conditions including attention deficit hyperactivity disorder (ADHD), obsessive compulsive disorder (OCD) and to a lesser extent autism spectrum disorder. ${ }^{3}$ In addition to the core clinical features that are integral to TS, a wide range of other behavioral, emotional, and psychiatric conditions have been reported to occur more commonly in TS as compared to the general population (eg, ADHD in $60 \%$ of the TS population as compared to $5 \%-10 \%$ in the general population and OCD in $30 \%-50 \%$ of the TS population as compared to $1 \%-3 \%$ in the general population) and previous studies have also suggested that increased tic severity is associated with increased psychopathology., ${ }^{4-11}$ Further, TS has been to a lesser extent associated with autism spectrum disorder. ${ }^{3,9,11}$ A number of co-existent psychopathologies have also been described in TS including anxiety, depression, learning difficulties, personality disorder, impulse control, aggression, oppositional defiant disorder, and conduct disorder. ${ }^{6}$ While the occurrence of the core clinical features and associated comorbid conditions have been found to be similar irrespective of country of origin, ${ }^{12}$ highlighting the biological nature of TS, there have been some differences noted between cultures with regard to the occurrence of associated psychopathologies, suggesting complex geneenvironment interactions. . $^{13,14}$

Although TS is known in the lay literature for coprophenomena (coprolalia and copropraxia), the prevalence rates in TS patients have been observed to vary considerably, ${ }^{15,16}$ with rates of coprolalia reported in $21 \%,{ }^{17} 25 \%,{ }^{18} 33 \%,{ }^{19}$ $42 \%,{ }^{20}$ and $43 \%,{ }^{21}$ and copropraxia in $7 \%,{ }^{18} 17 \%,{ }^{19} 21 \%,{ }^{17}$ and $23 \%{ }^{21}$ of individuals with TS. It is also to be noted that coprophenomena are not unique to tic disorders and their occurrence has been reported following stroke, frontotemporal dementia, encephalitis, epilepsy, Lesch-Nyhan syndrome, and choreoacanthocytosis..$^{22-24}$ In these instances of adult-onset tic disorders, there is often a potential trigger such as a neurological event with a temporal relationship to the onset of the symptoms, the symptoms are more severe with greater social morbidity, increased sensitivity, and poorer response to neuroleptic medication. ${ }^{24}$

A multi-site collaborative study undertaken by the Tourette Syndrome International Database Consortium involving a subset of 597 patients from 15 sites in seven countries, reported that coprolalia occurred at some time in $19 \%$ of males and $15 \%$ of females, and copropraxia in $6 \%$ of males and $5 \%$ of females. ${ }^{25}$ Coprolalia was three times as frequent as copropraxia, with a mean onset of each at about 11 years, 5 years after the onset of tics. However, in $11 \%$ of those with coprolalia and $12 \%$ of copropraxia, these symptoms were one of the initial symptoms of TS. ${ }^{25}$ The most robust associations of coprophenomenon were with the number of non-tic repetitive behaviors, spitting and inappropriate sexual behavior. This suggested that the course and impact are variable and that more research is required. ${ }^{25}$ Given the varying results obtained in previous research assessing complex tics including the coprophenomenon, there is a need to better understand the prevalence and phenomenology of simple versus complex motor and vocal tics and associated phenomena. Furthermore, there is a need to evaluate the differences between individuals with pure-TS and TS-plus and simple versus complex tics. This study aimed to address this gap in knowledge.

\section{Methods \\ Participants}

A cohort of 400 patients attending specialized Tourette clinics in the UK fulfilling diagnostic criteria for TS was recruited for the study. A diagnosis of TS and any additional psychiatric diagnoses were made using either the DSM-111 to DSM-IV-TR ${ }^{26}$ edition of the Diagnostic and Statistical Manual for Mental Disorders diagnostic criteria, depending on when the patients were assessed. Patients were clinically assessed and examined by the second author, an experienced neuropsychiatrist (MMR) using standardized schedules. The mean age of the sample was 27.7 years and $72 \%$ were males. See Table 1 for additional patient characteristics including sex and age distribution.

Table I Demographic information of the study sample

\begin{tabular}{ll}
\hline $\mathrm{N}$ & 400 \\
$\mathrm{Sex}$ & $72 \%$ male \\
Mean age in years (SD: range) & 27.7 (I3.2: 8-78) \\
Mean YGTSS total score (SD: range) & 48.7 (I8.2: 5-94) \\
Mean DCI total score & 63.7 (I7.0: 16-100) \\
\hline
\end{tabular}

Abbreviations: YGTSS, Yale Global Tic Severity Scale; DCI, Diagnostic Confidence Index; SD, standard deviation. 
A subgroup of 222 TS patients who had full data on comorbidities and associated psychopathologies was selected for further analysis. Within this sample, individuals with pure-TS (motor and vocal tics only) were compared with participants who reported associated comorbidities (TS-plus).

\section{Measures}

Each participant underwent a one-time detailed clinical interview using three published schedules for assessing individuals with TS including the National Hospital Interview Schedule (NHIS) for TS and related behaviours, ${ }^{27}$ the Diagnostic Confidence Index (DCI), ${ }^{28}$ and the Yale Global Tic Severity Scale (YGTSS). ${ }^{29}$ The NHIS provides a standardized current diagnosis of TS characterized by multiple motor (more than one) and at least one vocal tic as per the Diagnostic and Statistical Manual for Mental Disorders criteria. The NHIS was used as a structured method for assessment of tics as present at interview, in the previous week and "ever" (lifetime) based on self-report for adults and self- and parent-reports in the case of children and they were also evaluated for related features, relevant family history, and the presence of comorbid conditions including OCD, ADHD, and any other co-existent psychopathology. The DCI provides a measure of whether an individual is likely to have met diagnostic criteria for TS in the past or currently. The YGTSS is a semi-structured clinician rated scale assessing tic severity based on the presence of tics in the previous week. Tics were classified as "simple" if it involved only a single group of muscles or a simple vocal/phonic sound. ${ }^{3}$ It is to be noted that invariably all patients who had complex tics also had simple tics.

\section{Data analysis}

Data were analyzed using SPSS Statistics version 22 (IBM Corporation, Armonk, NY, USA), with alpha set at 0.05 for all comparisons. Relationships between levels of categorical data were examined using Fisher's exact test, with phi values included to indicate the size of effects. For phi, values of 0.1 , 0.3 , and 0.5 are understood to denote small, medium, and large effects, respectively. Independent-samples Student's $t$-tests were conducted to explore group differences in continuous data, with Cohen's $d$ effect sizes also included. Cohen's $d$ values of $0.2,0.5$, and 0.8 denote small, medium, and large effects respectively.

Data were analyzed with the aim of examining the occurrences and inter-relationships between pure-TS (motor and vocal tics only) and TS-plus (tics associated with other comorbid conditions) and also between those with simple motor and vocal tics compared to those with complex motor and vocal tics including echophenomenon and complex vocalizations including coprophenomenon.

\section{Results}

Of the 400 participants, 156 (39\%) had experienced coprolalia while $79(20 \%)$ reported having had copropraxia. Independent $t$-tests revealed a significant difference between participants experiencing coprolalia and those who were not on both the YGTSS total score $t(326)=-5.9, P=<0.0001$, Cohen's $d=0.66$; and the DCI total score $t(214)=-11.23$, $P=<0.0001$, Cohen's $d=1.52$. In both instances, participants with coprolalia obtained higher scores. A significant relationship was also observed between the presence of coprolalia and the presence of copropraxia (Fisher's exact test, $P=<0.0001, \varphi=0.44$ ) as well as echolalia (Fisher's exact test, $P=<0.0001, \varphi=0.35$ ) with a higher proportion of participants with coprolalia also experiencing both copropraxia and echolalia. There were no significant associations between coprophenomena and comorbidities.

In the subset of 222 subjects where comorbidity data were available, $13.5 \%$ reported tics in the absence of comorbid disorder (pure-TS group) and the remainder (86.5\%) reported various comorbidities (TS-plus group). Independent $t$-tests revealed no significant differences between participants in the pure-TS group and TS-plus groups on either the YGTSS total score $t(165)=0.08, P=0.93$, Cohen's $d=0.02$; or the level of diagnostic confidence as indicated by the DCI total score $t(117)=-1.70, P=0.093$, Cohen's $d=0.50$. It can be noted that results from the DCI nonetheless approached significance, and the effect size in that instance was of medium size (see Table 2). The presence of coprolalia was higher (27.8\%) in those with TS-plus as compared to those with pure-TS $(0 \%)$ using the DCI and the difference in proportions was statistically significant (Fisher's exact test, $P=0.036, \varphi=0.20$ ). Similarly the presence of copropraxia was higher (15.4\%) in the TS-plus group as compared to the pure-TS (6.3\%) group. Although this did not reach statistical significance due to low experimental power resulting from the small number of subjects, the relative risk of coprolalia in TS-plus relative to pure-TS was found to be 1.5 , with a relative risk of copropraxia of 2.4. Further, individuals with coprolalia were found to have a significantly higher diagnostic confidence score $t(115)=-8.9, P<0.0001$, Cohen's $d=2.0$; and higher YGTSS total tic severity score that approached statistical significance $t(68)=-1.74, P=0.086$, Cohen's $d=0.47$. Thus there were significant correlations between the three measures utilized in the study using Spearman's rho (see Table 3). 
Table 2 Pure-TS group and TS-plus groups on the YGTSS and DCl scores

\begin{tabular}{lllll}
\hline & TS with and without comorbidities & Mean & SD & Cohen's d effect size \\
\hline YGTSS total & TS only & 53.39 & 18.98 & 0.02 \\
DCl total & TS + comorbidities & 53.06 & 17.77 & \\
& TS only & 55.23 & 18.67 & 0.50 \\
\hline
\end{tabular}

Abbreviations: YGTSS, Yale Global Tic Severity Scale; DCl, Diagnostic Confidence Index; SD, standard deviation; TS, Tourette syndrome.

No differences were found between the pure-TS and TS-plus groups in any of the other core features including echophenomenon (echolalia and echopraxia), and/or palilalia, palipraxia, family history of tics, or comorbidity etc. The only exception was that the pure-TS group was associated with no family history of OCD (Fisher's exact test, $P<0.0001, \varphi=0.34)$.

Analysis of those patients with only simple tics (motor and vocal) as compared to complex motor behaviors and vocalizations revealed that there were no significant differences in any of the core clinical features, or with regards to severity, or level of diagnostic confidence. However, those with complex motor/vocal tics were significantly more likely to report a premonitory urge or sensation (Fisher's exact test, $P=0.006, \varphi=0.15$ ).

\section{Discussion}

Our finding that only $13.5 \%$ of TS patients have pure motor and vocal tics only (ie, pure-TS) is in keeping with previous international studies that have reported that around $10 \%$ of TS patients have only tics in both clinical ${ }^{25}$ and community ${ }^{30,31}$ settings. The rate of coprolalia in our cohort was $39 \%$ which is higher than the previously reported $20 \%$ from an international database, ${ }^{25}$ but similar to previous cohorts from the same clinic which may be a reflection of the referral bias to this specialized TS clinic. ${ }^{20,21}$ Our finding that the DCI scores did not differ in those with pure-TS versus TS-plus suggests that the current diagnostic criteria are an accurate way of defining the core clinical syndrome of TS. ${ }^{13}$ Further, we did not find any differences between individuals who exhibited simple motor or vocal tics as compared to those with complex movements and vocalizations.
We observed a significant association between complex motor and vocal tics and the presence of premonitory urges. This may at least in part be due to the fact that patients may be more aware of complex tics (motor and vocal) when they occur and hence may have been more perceptive of the premonitory urges that precede these tics. It may also be because of the activation of a particular neurophysiological component linked to a specific neuronal circuitry in the genesis of complex tics which also results in premonitory urges. In this regard, it has been observed that slightly different psychological factors drive self-reported premonitory urge ratings in patients with and without comorbidities ${ }^{32}$ and that different types of premonitory urges are associated with simple/complex tics and obsessive-compulsive symptoms. ${ }^{33}$ Premonitory urges are an important symptom, occurring in about $90 \%$ of TS patients ${ }^{34,35}$ and are usually identified by patients after the age of 10 years. ${ }^{11}$ The relationship between the premonitory urges and tic severity is inconsistent, with some studies showing no association ${ }^{36,37}$ and others indicating a positive relationship..$^{38-41}$ It is plausible that the conflicting results observed in different studies may be a reflection of the proportion of subjects in the respective cohorts with complex motor/vocal tics as well as comorbidities.

Our findings of an increased occurrence of coprophenomenon in TS-plus cases, coupled with an association found between coprolalia and higher YGTSS and DCI scores, is in keeping with a recent study that reported that coprophenomenon occurred when tics are most severe and was associated with comorbidity. ${ }^{18}$ Thus it appears that, while there are no significant differences between the types of tics per se (ie, simple or complex tics) in terms of severity or associated clinical features, the presence of coprophenomenon may

Table 3 Spearman's rho correlations between coprolalia, severity of symptoms, and diagnostic confidence

\begin{tabular}{llll}
\hline & Coprolalia & YGTSS severity score & Diagnostic confidence index \\
\hline Coprolalia & $\mathrm{I}$ & & \\
YGTSS severity score & $0.31^{*}$ & $\mathrm{I}$ & $\mathrm{I}$ \\
Diagnostic confidence index & $0.60^{*}$ & $0.42^{*}$ & \\
\hline
\end{tabular}

Note: $* P<0.01$.

Abbreviation: YGTSS, Yale Global Tic Severity Scale. 
indicate a more severe form of the disorder and perhaps more extensive involvement of the cortico-striatal-thalamocortical circuitry implicated in TS. ${ }^{22}$ For example, striatal and basal ganglia site of the circuitry may be involved in tics only, while the involvement of the frontal cortical end of the circuitry may result in more socially inappropriate behaviors. ${ }^{4}$ Similarly, the presence of comorbidities may suggest additional circuitry being involved such as the basal ganglia-limbic circuits in OCD and so forth which in turn may point to the involvement of additional genetic or environmental factors. ${ }^{42}$ The finding in the current study that pure-TS was associated with no family history of OCD seems to suggest this. It could be postulated that, in addition to specific TS gene(s) that are involved in the development of motor and vocal tics in pure-TS, there may be additional genes (eg, linked to OCD) and/or environmental factors involved when TS is associated with other comorbidities and psychopathologies.

While there is little doubt that the vulnerability to developing tics is genetically determined, there are a number of other genetic, epigenetic, and environmental factors that contribute to the final clinical expression. The multi-strike model $^{43}$ suggests that the location and extent of involvement of the circuitry may also be determined by a multitude of factors. In this model, initial genetic vulnerability (or the first strike) results in specific structural and/or functional changes in key brain areas and predisposes the individual to develop tics and related symptoms. The second strike causes disturbances in the neuronal circuitry and neurotransmitter levels in the brain regions that would normally support selfregulatory compensatory responses that serve to inhibit tic expression. This results in the development of tic symptoms. The third strike, that may occur through a number of other pre- and post-natal events, would exert its influence in determining the final clinical expression of TS including the severity and comorbidities. ${ }^{44}$ Thus, it may be that different sites of the circuitry may be affected based on the different time points in the development (intrauterine and postnatally) when the second or third strike is occurring as different brain regions are formed during intrauterine development and then mature postnatally at different time points. There would also be a number of other modulating factors including sex with the suggested sex-dependent difference in the phenotypic expression of the putative TS genes with males showing tic related behaviors and females exhibiting obsessive compulsive behaviours. ${ }^{45}$ Future studies should therefore explore the genetic and phenotypic relationship in conjunction with environmental variables to account for the contribution of these factors in clinical presentation and severity to better understand the pathogenesis in pure-TS versus TS-plus.

While the large sample size and detailed evaluation of patients using standardized instruments by experienced specialists with expertise in TS add to the strength of this study, there are a number of limitations that would merit highlighting. In this regard, it is to be noted that this was a naturalistic cohort study of patients who attended a specialized TS clinic and that the assessor was not blind to the group status as to whether the participants belonged to the pure-TS versus TSplus group. Further, the higher rates of coprophenomenon observed in this study may be a reflection of the referral bias to this tertiary clinic and this might limit the generalizability of our findings to other settings. However, the finding that the majority of TS patients have associated comorbidities and psychopathologies suggests the need for comprehensive evaluation of TS patients. Further, it would appear that while those with pure-TS and having only simple motor and vocal tics may only need psycho-education, those with TS-plus and associated complex tics and coprophenomenon would require a combination of psychological and pharmacological intervention to address the tics as well as the associated psychopathologies. Thus, the detailed assessment of tics as well as the associated conditions has significant implications for the management of TS as early recognition of the associated comorbidities and co-existent psychopathologies can improve the overall outcome and quality of life in TS.

\section{Disclosure}

The authors have no conflicts of interest to disclose.

\section{References}

1. World Health Organization. International Classification of Diseases, Tenth edition (ICD-10). Geneva, Switzerland: World Health Organization; 1992.

2. American Psychiatric Association. Diagnostic and Statistical Manual of Mental Disorders - Fifth Edition (DSM-5). Washington, DC: American Psychiatric Publishing; 2013.

3. Eapen V, McPherson S, Sneddon C, Črnčec R. Tic disorders and learning disability: Clinical characteristics, cognitive performance and co-morbidity. Australasian Journal of Special Education. 2013;37(2):162-172.

4. Robertson MM, Eapen V. Wither the relationship between etiology and phenotype in Tourette Syndrome? In: Martino D, Leckman JF, editors. Tourette Syndrome. New York: Oxford University Press Inc; 2013: 361-394.

5. Freeman RD, Fast DK, Burd DL, Kerbashian J, Robertson MM, Sandor P. An international perspective on Tourette syndrome: selected findings from 3500 individuals in 22 countries. Dev Med Child Neurol. 2000;42(7):436-447.

6. Robertson MM, Cavanna AE, Eapen V. Gilles de la Tourette Syndrome and disruptive behavior disorders: Prevalence, associations and explanation of the relationships. J Neuropsychiatry Clin Neurosci. Epub 2014 Aug 27.

7. Singer HS. Tourette's syndrome: from behaviour to biology. Lancet Neurol. 2005;4(3):149-159. 
8. Leckman JF. Tourette's syndrome. Lancet. 2002;360(9345): $1577-1586$.

9. Robertson MM. The Gilles de la Tourette Syndrome: The current status. Arch Dis Child Educ Pract Ed. 2012;97(5):166-175.

10. Robertson MM. Gilles de la Tourette syndrome: the complexities of phenotype and treatment. Br J Hosp Med (Lond). 2011;72(2):100-107.

11. Eapen V, Črnčec R. Tourette syndrome in children and adolescents: Special considerations. J Psychosom Res. 2009;67(6):525-532.

12. Robertson MM. The prevalence and epidemiology of Gilles de la Tourette syndrome. Part 1: the epidemiological and prevalence studies. J Psychosom Res. 2008;65(5):461-472.

13. Robertson MM, Eapen V. Tourette's: Syndrome, Disorder or Spectrum? Classificatory Challenges and an appraisal of the DSM criteria. Asian Journal of Psychiatry. In press 2014.

14. Eapen V, Robertson MM. Clinical correlates of Tourette's disorder across cultures: A comparative study between the United Arab Emirates and the United Kingdom. Prim Care Companion J Clin Psychiatry. 2008;10(2):103-107.

15. Miranda M, Menendez P, David P, Troncoso M, Hernández M, Chaná P. [Tics disease (Gilles de la Tourette syndrome): Clinical characteristics of 70 patients]. Rev Med Chil. 1999;127(12):1480-1486. Spanish.

16. Kano Y, Ohta M, Nagai Y. Differences in clinical characteristics between Tourette syndrome patients with and without 'generalized tics' or coprolalia. Psychiatry Clin Neurosci. 1997;51(6):357-361.

17. Lees AJ, Robertson M, Trimble MR, Murray NM. A clinical study of Gilles de la Tourette syndrome in the United Kingdom. $J$ Neurol Neurosurg Psychiatry. 1984;47(1):1-8.

18. Kobierska M, Sitek M, Gocyła K, Janik P. Coprolalia and copropraxia in patients with Gilles de la Tourette syndrome. Neurol Neurochir Pol. 2014;48(1):1-7.

19. Robertson MM, Trimble MR, Lees AJ. The psychopathology of the Gilles de la Tourette syndrome. A phenomenological analysis. $\mathrm{Br} J$ Psychiatry. 1988;152:383-390.

20. Rickards H, Robertson M. A controlled study of psychopathology and associated symptoms in Tourette syndrome. World J Biol Psychiatry. 2003;4(2):64-68.

21. Eapen V, Fox-Hiley P, Banerjee S, Robertson MM. Tourette's Syndrome: A clinical and psychopathological study in an adult UK cohort. Acta Neurol Scand. 2004;109(4):255-260.

22. Eapen N, Yakely JW, Robertson MM. Obsessive-compulsive disorder and self-injurious behavior. In: Kurlan R, editor. Handbook of Tourette's and related tic and behavioral disorders. 2nd ed. New York: Marcel Dekker; 2005:39-88.

23. Jankovic J, Kwak C. Tics in other neurological disorders. In: Kurlan R, editor. Handbook of Tourette's and related tic and behavioral disorders. 2nd ed. New York: Marcel Dekker; 2005:173-194.

24. Eapen V, Lees AJ, Lakke JP, Trimble MR, Robertson MM. Adult-onset tic disorders. Mov Disord. 2002;17(4):735-740.

25. Freeman RD, Zinner SH, Muller-Vahl KR, et al. Coprophenomena in Tourette syndrome. Dev Med Child Neurol. 2009;51(3):218-227.

26. American Psychiatric Association. Diagnostic and Statistical Manual of Mental Disorders (DSM-IV). 4th ed. Washington, DC: American Psychiatric Publishing; 2000.
27. Robertson MM, Eapen V. The National Hospital Interview Schedule for the assessment of Gilles de la Tourette syndrome. Int J Meth Psychiatr Res. 1996;6:203-226.

28. Robertson MM, Banerjee S, Kurlan R, et al. The Tourette Diagnostic Confidence Index: Development and clinical associations. Neurology. 1999;53(9):2108-2112.

29. Leckman JF, Riddle MA, Hardin MT, et al. The Yale Global Tic Severity Scale: Initial testing of a clinician-rated scale of tic severity. $J \mathrm{Am}$ Acad Child Adolesc Psychiatry. 1989;28(4):566-573.

30. Eddy CM, Cavanna AE. It's a 'curse!': coprolalia in Tourette Syndrome. Eur J Neurol. 2013;20(11):1467-1470.

31. Robertson MM, Cavanna AE, Eapen V. Gilles de la Tourette syndrome and disruptive behaviour disorders: Prevalence, associations and explanations of the relationships. $J$ Neuropsychiatry Clin Neurosci. Epub 2014 Aug 27.

32. Eddy CM, Cavanna AE. Premonitory urges in adults with complicated and uncomplicated Tourette Syndrome. Behav Modif. 2013;38(2):264-275.

33. Rajagopal S, Cavanna AE. Premonitory urges and repetitive behaviours in adult patients with Tourette syndrome. Neurol Sci. 2014;35(6): 969-971.

34. Leckman JF, Walker DE, Cohen DJ. Premonitory urges in Tourette's syndrome. Am J Psychiatry. 1993;150(1):98-102.

35. Kwak C, Dat Vuong K, Jankovic J. Premonitory sensory phenomenon in Tourette's syndrome. Mov Disord. 2003;18(12):1530-1533.

36. Steinberg T, Shmuel Baruch S, Harush A, et al. Tic disorders and the premonitory urge. J Neural Transm. 2010;117(2):277-284.

37. Sutherland Owens AN, Miguel EC, Swerdlow NR. Sensory gating scales and premonitory urges in Tourette syndrome. Scientific World Journal. 2011;11:736-741.

38. Crossley E, Cavanna AE. Sensory phenomena: clinical correlates and impact on quality of life in adult patients with Tourette syndrome. Psychiatry Res. 2013;209(3):705-710.

39. Woods DW, Piacentini J, Himle MB, Chang S. Premonitory Urge for Tics Scale (PUTS): initial psychometric results and examination of the premonitory urge phenomenon in youths with Tic disorders. $J$ Dev Behav Pediatr. 2005;26(6):397-403.

40. Reese HE, Scahill L, Peterson AL, et al. The premonitory urge to tic: measurement, characteristics, and correlates in older adolescents and adults. Behav Ther. 2014;45(2):177-186.

41. Crossley E, Seri S, Stern JS, Robertson MM, Cavanna AE. Premonitory urges for tics in adult patients with Tourette syndrome. Brain Dev. 2014;36(1):45-50.

42. Eapen V. Neurodevelopmental Genes Have Not Read The DSM Criteria: Or, Have They? Front Psychiatry. 2012;23:75.

43. Spessot AL, Plessen KJ, Peterson BS. Neuroimaging of developmental psychopathologies: the importance of self-regulatory and neuroplastic processes in adolescence. Ann N Y Acad Sci. 2004;1021:86-104.

44. Gorman DA, Abi-Jaoude E. Uncovering the complexity of Tourette syndrome, little by little. Br J Psychiatry. 2014;204(1):6-8.

45. Eapen V, Pauls DL, Robertson MM. Evidence for autosomal dominant transmission in Tourette's syndrome. United Kingdom cohort study. Br J Psychiatry. 1993;162:593-596.
Neuropsychiatric Disease and Treatment

\section{Publish your work in this journal}

Neuropsychiatric Disease and Treatment is an international, peerreviewed journal of clinical therapeutics and pharmacology focusing on concise rapid reporting of clinical or pre-clinical studies on a range of neuropsychiatric and neurological disorders. This journal is indexed on PubMed Central, the 'PsycINFO' database and CAS,

\section{Dovepress}

and is the official journal of The International Neuropsychiatric Association (INA). The manuscript management system is completely online and includes a very quick and fair peer-review system, which is all easy to use. Visit http://www.dovepress.com/testimonials.php to read real quotes from published authors. 